\title{
Measurement of vector boson plus photon production with the ATLAS detector and associated constraints on new physics
}

\author{
Louis HELARY*广 \\ Boston University \\ E-mail: louis.helarydcern.ch
}

\begin{abstract}
We present the measurement of the integrated and differential fiducial cross sections for the production of a $W$ or $Z$ boson in association with a high energy photon, using ATLAS pp collision data at $\sqrt{s}=7 \mathrm{TeV}$ and an integrated luminosity of $\int \mathscr{L} d t=4.6 \mathrm{fb}^{-1}$. Events are selected using leptonic decays of the $W(W(e v, \mu v))$ and $Z(Z(e e, \mu \mu, v v))$ bosons. The data are used to test the Electroweak sector of the Standard Model and search for evidence of new phenomena. The measurements are used to probe anomalous triple gauge boson couplings and to search for the production of vector resonances decaying to $Z \gamma$ and $W \gamma$.
\end{abstract}

The European Physical Society Conference on High Energy Physics -EPS-HEP2013

18-24 July 2013

Stockholm, Sweden

\footnotetext{
${ }^{*}$ Speaker.

†n the behalf of the ATLAS collaboration.
} 


\section{Introduction}

The study [1] of the $W \gamma$ and $Z \gamma$ final states consitutes an important test of the Standard Model $(\mathrm{SM})$. These states allow one to measure with precision the triple gauge couplings predicted by the non-Abelian $S U(2)_{L} \times U(1)_{Y}$ gauge group of the Electroweak sector. Any deviation from the SM prediction could be a sign of new physics. The measurements presented here were obtained using full dataset of $p p$ collision at a center-of-mass energy of $\sqrt{s}=7 \mathrm{TeV}$ collected in 2011 by the ATLAS detector, corresponding to an integrated luminosity of $\int \mathscr{L} d t=4.6 \mathrm{fb}^{-1}$. The $W \gamma$ and $Z \gamma$ final states are studied using the production processes $p p \rightarrow \ell v \gamma+X, p p \rightarrow \ell^{+} \ell^{-} \gamma+X$ and $p p \rightarrow v \bar{v} \gamma+X$, where $(\ell=e, \mu)$. This note is organized as follows: first the event selection and background estimation are introduced; then, the fiducial and differential cross-section measurements are presented; finally, the study of these final states is used to extract limits on new physics.

\section{Selection and background estimation}

\subsection{The $\ell v \gamma$ final state}

The $\ell v \gamma$ events are selected if they contain exactly one isolated electron or muon with $p_{\mathrm{T}}^{\ell}>$ $25 \mathrm{GeV}$, at least one isolated photon with $E_{\mathrm{T}}^{\gamma}>15 \mathrm{GeV}$ and missing transverse energy $E_{\mathrm{T}}^{\text {miss }}>$ $35 \mathrm{GeV}$. Furthermore, the transverse mass ${ }^{1}$ of the Lepton- $E_{\mathrm{T}}^{\text {miss }}$ system is required to be $m_{\mathrm{T}}>$ $40 \mathrm{GeV}$. The lepton and the photon are required to be in the pseudorapidity range covered by the inner-tracker $|\eta| \lesssim 2$.4. In the electron channel, a $Z$-veto is applied by requiring that the electronphoton invariant mass $\left(m_{e \gamma}\right)$ is not within $15 \mathrm{GeV}$ of the $Z$ boson mass; this cut is applied to reduce the $Z \rightarrow e^{+} e^{-}$background. The lepton and photon must be separated by a distance ${ }^{2}$ of $\Delta R(\ell, \gamma)>0.7$ to reduce the final state radiation contribution. In the following, jets are considered if $p_{\mathrm{T}}^{\text {jet }}>30 \mathrm{GeV}$ and $\left|\eta^{\text {jet }}\right|<4.4$. The main background processes for $\ell v \gamma$ events are $W(\ell v)+$ jets, $Z \rightarrow \ell \ell, \gamma+$ jets and $t \bar{t}$. The event yield and template shape for the $W+$ jets and $\gamma+$ jets processes are estimated from data-driven techniques. All other backgrounds are estimated from MC simulations. The purity of $\ell v \gamma$ events after selection is found to be approximately $60 \%$.

\subsection{The $\ell^{+} \ell^{-} \gamma$ final state}

The $\ell^{+} \ell^{-} \gamma$ events are required to have at least one isolated photon with $E_{\mathrm{T}}^{\gamma}>15 \mathrm{GeV}$ and two same-flavour opposite-charge leptons with $p_{\mathrm{T}}^{\ell}>25 \mathrm{GeV}$. The invariant mass of the dilepton system must be $m_{\ell \ell}>40 \mathrm{GeV}$. The spatial separation between the leptons and the photon should be the same as in the $\ell v \gamma$ final state $(\Delta R(\ell, \gamma)>0.7)$. As for the $\ell v \gamma$ final state, jets are considered if $p_{\mathrm{T}}^{\text {jet }}>30 \mathrm{GeV}$ and $\left|\eta^{\text {jet }}\right|<4$.4. Two major backgrounds contribute to this final state: $Z \rightarrow \ell \ell+$ jets and $t \bar{t}$. The $Z \rightarrow \ell \ell+$ jets event shape and yield are completely determined from the data, while the remaining backgrounds are estimated from MC simulation. The event purity is found to be approximately $85 \%$ for this final state.

\footnotetext{
${ }^{1} m_{\mathrm{T}}=\sqrt{2 p_{\mathrm{T}}(\ell) \times E_{\mathrm{T}}^{\text {miss }} \times(1-\cos \Delta \phi)}$, where $\Delta \phi$ represents the difference between the azimutal angle of the lepton and the missing transverse energy.

${ }^{2}$ The distance $\Delta R$ in the $\eta-\phi$ space is defined as $\Delta R=\sqrt{(\Delta \eta)^{2}+(\Delta \phi)^{2}}$.
} 


\subsection{The $v \bar{v} \gamma$ final state}

Events are considered in the $v \bar{v} \gamma$ analysis if they contain one isolated photon with $E_{\mathrm{T}}^{\gamma}>100$ $\mathrm{GeV}$ as well as missing transverse energy $E_{\mathrm{T}}^{\text {miss }}>90 \mathrm{GeV}$, the two must be well separated in the transverse plan: $\Delta \phi\left(E_{\mathrm{T}}^{\mathrm{miss}}, \gamma\right)>2.6$. Once again, a jet is considered if $p_{\mathrm{T}}^{\text {jet }}>30 \mathrm{GeV}$ and $\left|\eta^{\text {jet }}\right|<$ 4.4. If jets are found in the event, they are required to be separated from the missing transverse energy: $\Delta \phi\left(E_{\mathrm{T}}^{\mathrm{miss}}\right.$, jet $)>0.4$. These angular cuts are applied to reduce the $\gamma+\mathrm{jets}$ background. The event is vetoed if any lepton is identified. The main background processes for the $v \bar{v} \gamma$ events are $W \gamma, W(e v), \gamma+$ jets and $Z(v \bar{v})+$ jets. The event yield for the $W(e v), Z(v \bar{v})+$ jets and $\gamma+$ jets events are estimated from data-driven techniques. The other backgrounds are estimated from MC simulation. The purity for the $v \bar{v} \gamma$ selection is found to be approximately $50 \%$.

\section{Cross-section measurement}

\subsection{Fiducial cross-section measurement}

The cross-sections of both the $W \gamma$ and $Z \gamma$ processes are evaluated in an extended fiducial volume defined in Table 1 and computed according to Equation 3.1.

$$
\sigma_{p p \rightarrow \ell v \gamma\left(\ell^{+} \ell^{-} \gamma / v \bar{v} \gamma\right)}^{\mathrm{ext}-\mathrm{fid}}=\frac{N_{V \gamma}^{\mathrm{sig}}}{A_{V \gamma} \cdot C_{V \gamma} \cdot \int \mathscr{L} d t .}
$$

In this equation, $N_{V \gamma}^{\mathrm{sig}}$ is the number of signal events obtained from the data-background subtraction; $A_{V \gamma}$ is the acceptance, defined as the number of events generated in the fiducial volume divided by the number of events generated in the extended fiducial volume; $C_{V \gamma}$ is the probability for an event generated in the fiducial volume to be reconstructed in the selection defined in Section 2; and $\int \mathscr{L} d t$ is the integrated luminosity $\left(4.6 \mathrm{fb}^{-1}\right)$.

\begin{tabular}{lccc}
\hline Cuts & $p p \rightarrow \ell v \gamma$ & $p p \rightarrow \ell^{+} \ell^{-} \gamma$ & $p p \rightarrow v \bar{v} \gamma$ \\
\hline Lepton & $p_{\mathrm{T}}^{\ell}>25 \mathrm{GeV}$ & $p_{\mathrm{T}}^{\ell}>25 \mathrm{GeV}$ & - \\
& $\left|\eta_{\ell}\right|<2.47$ & $\left|\eta_{\ell}\right|<2.47$ & - \\
& $N_{\ell}=1$ & $N_{\ell^{+}}=1, N_{\ell^{-}}=1$ & $N_{\ell}=0$ \\
\hline Neutrino & $p_{\mathrm{T}}^{v}>35 \mathrm{GeV}$ & - & - \\
\hline Boson & - & $m_{\ell^{+} \ell^{-}}>40 \mathrm{GeV}$ & $p_{\mathrm{T}}^{v \bar{v}}>90 \mathrm{GeV}$ \\
\hline Photon & $E_{\mathrm{T}}^{\gamma}>15 \mathrm{GeV}$ & $E_{\mathrm{T}}^{\gamma}>15 \mathrm{GeV}$ & $E_{\mathrm{T}}^{\gamma}>100 \mathrm{GeV}$ \\
& $\left|\eta^{\gamma}\right|<2.37, \Delta R(\ell, \gamma)>0.7$ \\
Jet & $\varepsilon_{h}^{p}<0.5$ \\
\multicolumn{4}{c}{$E_{\mathrm{T}}^{\text {jet }}>30 \mathrm{GeV},\left|\eta^{\text {jet }}\right|<4.4$} \\
$\Delta R(e / \mu / \gamma$, jet $)>0.3$ \\
Inclusive $: N_{\text {jet }} \geq 0$, Exclusive $: N_{\text {jet }}=0$
\end{tabular}

Table 1: Definition of the extended fiducial region where the cross sections are evaluated; $p_{\mathrm{T}}^{v}$ is the transverse momentum of the neutrino from $W$ decays; $p_{\mathrm{T}}^{v \bar{v}}$ is the transverse momentum of the $Z$ boson that decays into two neutrinos; $N_{\ell}$ is the number of leptons in one event; $\varepsilon_{h}^{p}$ [2] is the truth photon isolation fraction.

The cross-sections measured in the extended fiducial volume for each channel are presented in Table 2. Two measurements are provided for each final state: an inclusive measurement where no requirement is made on the jet multiplicity and an exclusive measurement where the events are required to have no energetic jets. The main sources of systematic uncertainty on the crosssections are due to the background estimate (10\% for $\ell v \gamma, 5 \%$ for $\ell^{+} \ell^{-} \gamma$ and for $15 \% v \bar{v} \gamma$ ), photon identification $(6 \%)$, and the uncertainty on the integrated luminosity $(4 \%)$. 


\begin{tabular}{ccc}
\hline & $\begin{array}{c}\sigma^{\text {ext-fid }}[\mathrm{pb}] \\
\text { Measurement }\end{array}$ & $\begin{array}{c}\sigma^{\text {ext-fid }}[\mathrm{pb}] \\
\text { MCFM Prediction }\end{array}$ \\
\hline \multicolumn{3}{c}{$N_{\text {jet }} \geq 0$} \\
\hline$\ell v \gamma$ & $2.77 \pm 0.03$ (stat) \pm 0.33 (syst) \pm 0.14 (lumi) & $1.96 \pm 0.17$ \\
$\ell^{+} \ell^{-} \gamma$ & $1.31 \pm 0.02$ (stat) \pm 0.11 (syst) \pm 0.05 (lumi) & $1.18 \pm 0.05$ \\
$v \bar{v} \gamma$ & $0.133 \pm 0.013$ (stat) \pm 0.020 (syst) \pm 0.005 (lumi) & $0.156 \pm 0.012$ \\
\hline \multicolumn{3}{c}{$N_{\text {jet }}=0$} \\
\hline$\ell v \gamma$ & $1.76 \pm 0.03$ (stat) \pm 0.21 (syst) \pm 0.08 (lumi) \\
$\ell^{+} \ell^{-} \gamma$ & $1.05 \pm 0.02$ (stat) \pm 0.10 (syst) \pm 0.04 (lumi) & $1.39 \pm 0.13$ \\
$\nu \bar{v} \gamma$ & $0.116 \pm 0.010$ (stat) \pm 0.013 (syst) \pm 0.004 (lumi) & $0.115 \pm 0.06 \pm 0.009$ \\
\hline
\end{tabular}

Table 2: Measured cross sections for the $\ell v \gamma, \ell^{+} \ell^{-} \gamma$ and $v \bar{v} \gamma$ processes at $\sqrt{s}=7 \mathrm{TeV}$ in the extended fiducial region defined in Table 1 . The statistical uncertainty for each measurement corresponds to the statistical uncertainty of the data sample used by the measurement. The SM predictions from MCFM [3], calculated at NLO, are also shown in the table with systematic uncertainties.

The measurements are compared to NLO predictions obtained with MCFM [3] interfaced to the СТ 10 [4] parton density function (pdf). In the $\ell^{+} \ell^{-} \gamma$ and $v \bar{v} \gamma$ final states the cross-sections measured in the data are found to be in good agreement with the NLO predictions. In the $\ell v \gamma$ final state the measurement is found to be slightly higher in the exclusive analysis and significantly higher in the inclusive analysis. MCFM provides real NLO prediction up to $\ell v \gamma+1$ parton. Therefore the discrepancy between the MCFM NLO predictions and the measurement in the $\ell v \gamma+X$ channel is essentially due to the significant contribution from multi-jet production, which is not included in these predictions. The measurement of the fiducial cross-section in the $v \bar{v} \gamma$ final state is the first measurement in this final state presented by the ATLAS collaboration.

\subsection{Differential cross-section measurement}

The cross-section measurement is also provided as a function of the $E_{\mathrm{T}}^{\gamma}$ spectrum for both the inclusive and exclusive analyses. Such measurements provide a more detailed comparison to the theoretical prediction since they allow for a direct and generic comparison at the particle level to any MC generator. The photon kinematic spectrum is unfolded using a Bayesian iterative unfolding technique. This technique is not sensitive to the MC simulation modeling of the spectrum shape. Furthermore one may choose the $E_{\mathrm{T}}^{\gamma}$ bins to be large compared to the detector resolution so as to minimize migration effects as well as to maintain a sufficient number of events in each bin.

Figure 1 shows the differential cross-section as a function of the photon $E_{\mathrm{T}}^{\gamma}$ for the (left) $p p \rightarrow \ell v \gamma$ and (right) $p p \rightarrow \ell^{+} \ell^{-} \gamma$ processes. In the $\ell v \gamma$ channel, the differential measurement is compared to the multi-leg LO generators SHERPA [5] and ALPGEN [6], interfaced to the CTEQ6.6M [7] and CTEQ6L1 [8] pdf respectively, scaled up by a global factor to match the total number of events observed in data. The MC predictions successfully reproduce the shape of the distribution measured in data. The measurement is also compared to the NLO MCFM predictions. In the inclusive analysis, the measurement shows a discrepancy with the NLO prediction at high $E_{\mathrm{T}}^{\gamma}$. In the exclusive analysis, the agreement between the NLO prediction and the measurement is improved. In the $\ell^{+} \ell^{-} \gamma$ final state, the differential measurement is compared to the SHERPA LO and MCFM NLO predictions. As for the $\ell v \gamma$ final state the SHERPA predictions are scaled up to match the total number of observed events. The ALPGEN generator does not provide a prediction for this final state and is therefore not used here. The SHERPA predictions also properly reproduce 
the $E_{\mathrm{T}}^{\gamma}$ spectrum in this final state. Comparisons of the MCFM NLO predictions to the measurement show a fair agreement for the inclusive analysis, and a good agreement in the exclusive analysis. The differential measurement presented in the $\ell^{+} \ell^{-} \gamma$ final state is the first to be realized by the ATLAS collaboration.
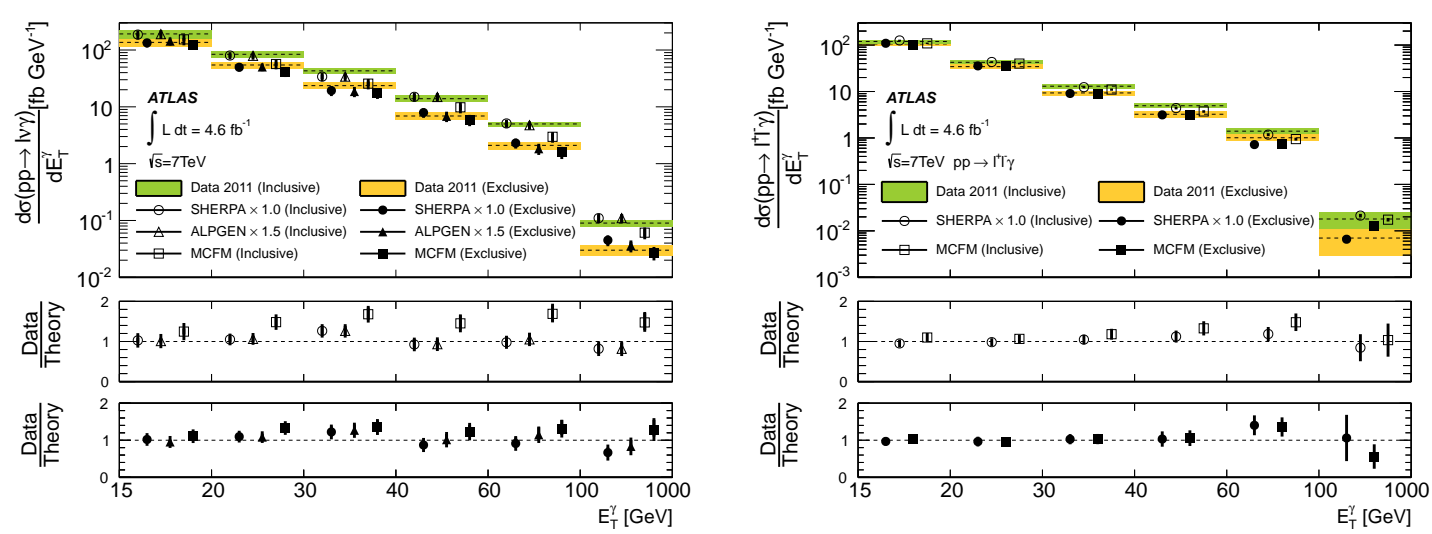

Figure 1: Measured $E_{\mathrm{T}}^{\gamma}$ differential cross sections [1] for the (left) $p p \rightarrow \ell v \gamma$ and (right) $p p \rightarrow \ell^{+} \ell^{-} \gamma$ processes, using combined electron and muon measurements in the inclusive $\left(N_{\text {jet }} \geq 0\right)$ and exclusive $\left(N_{\text {jet }}=0\right)$ extended fiducial regions. The lower plots show the ratio of the data to the predictions by different generators. The Monte Carlo uncertainties are shown only in the ratio plots. The cross-section predictions of the SHERPA and ALPGEN generators have been scaled by a global factor to match the total number of events observed in data. The global factor is 1.5 for the ALPGEN $\ell v \gamma$ signal sample and 1.0 for the SHERPA $\ell^{+} \ell^{-} \gamma$ signal sample. No global factor is applied for MCFM predictions.

\section{Constraint on new physics}

Using the precise determination of the $\ell v \gamma, \ell^{+} \ell^{-} \gamma$ and $v \bar{v} \gamma$ final states, searches for beyond SM physics are performed.

\subsection{Anomalous triple gauge coupling}

The high energy photon spectrum from $W \gamma$ and $Z \gamma$ events are sensitive to new phenomena that alter the couplings among the gauge bosons. The anomalous Triple Gauge Couplings (aTGCs) can be described using an effective Lagrangian. In order to avoid unitarity violation at high energy, form factors are introduced. Table 3 summarize the aTGCs [2] and the form factor used for each vertex.

\begin{tabular}{ccccc}
\hline Vertex & \multicolumn{2}{c}{ aTGC } & \multicolumn{2}{c}{ Form Factor } \\
\hline$W W \gamma$ & $\lambda_{\gamma}$ & $\Delta \kappa_{\gamma}$ & $\lambda_{\gamma}(s)=\lambda_{\gamma} /\left(1+\hat{s} / \Lambda^{2}\right)^{2}$ & $\Delta \kappa_{\gamma}(s)=\Delta \kappa_{\gamma} /\left(1+\hat{s} / \Lambda^{2}\right)^{2}$ \\
$Z Z \gamma$ & $h_{3}^{Z}$ & $h_{4}^{Z}$ & $h_{3}^{Z}(s)=h_{3}^{Z} /\left(1+\hat{s} / \Lambda^{2}\right)^{3}$ & $h_{4}^{Z}(s)=h_{4}^{Z} /\left(1+\hat{s} / \Lambda^{2}\right)^{4}$ \\
$Z \gamma \gamma$ & $h_{3}^{\gamma}$ & $h_{4}^{\gamma}$ & $h_{3}^{\gamma}(s)=h_{3}^{\gamma} /\left(1+\hat{s} / \Lambda^{2}\right)^{3}$ & $h_{4}^{\gamma}(s)=h_{4}^{\gamma} /\left(1+\hat{s} / \Lambda^{2}\right)^{4}$ \\
\hline
\end{tabular}

Table 3: aTGCs [2] and the form factor used for each vertex, where $\left(\Delta \kappa_{\gamma}=\kappa_{\gamma}-1\right), \sqrt{\hat{s}}$ is the $W \gamma$ or $Z \gamma$ invariant mass and $\Lambda$ is the new-physics energy scale.

A deviation of the aTGCs from the SM predictions would lead to an excess of events at high photon energy in these final states. Therefore the search for aTGCs is performed using the exclusive extended fiducial cross-sections for $W \gamma$ and $Z \gamma$ events with $E_{\mathrm{T}}^{\gamma}>100 \mathrm{GeV}$. The cross-section 
predictions with aTGCs $\left(\sigma_{W \gamma}^{\mathrm{aTGC}}\right.$ and $\left.\sigma_{Z \gamma}^{\mathrm{aTGC}}\right)$ are obtained from the MCFM generator. The limits on a given aTGC parameter are extracted from a Frequentist Profile Likelihood test, given the extended fiducial measurements. In the $Z \gamma$ channel, the $\ell^{+} \ell^{-} \gamma$ and the $v \bar{v} \gamma$ measurements are combined together to improve the search sensitivity. To conserve unitarity, the scale $\Lambda$ is chosen as $6 \mathrm{TeV}$ in the $W \gamma$ analysis and $3 \mathrm{TeV}$ in the $Z \gamma$ analysis. Results with an energy cut-off of $\Lambda=\infty$ are also presented as a comparison to the unitarity violation scheme. All the limits obtained in this analysis are presented on Table 4.

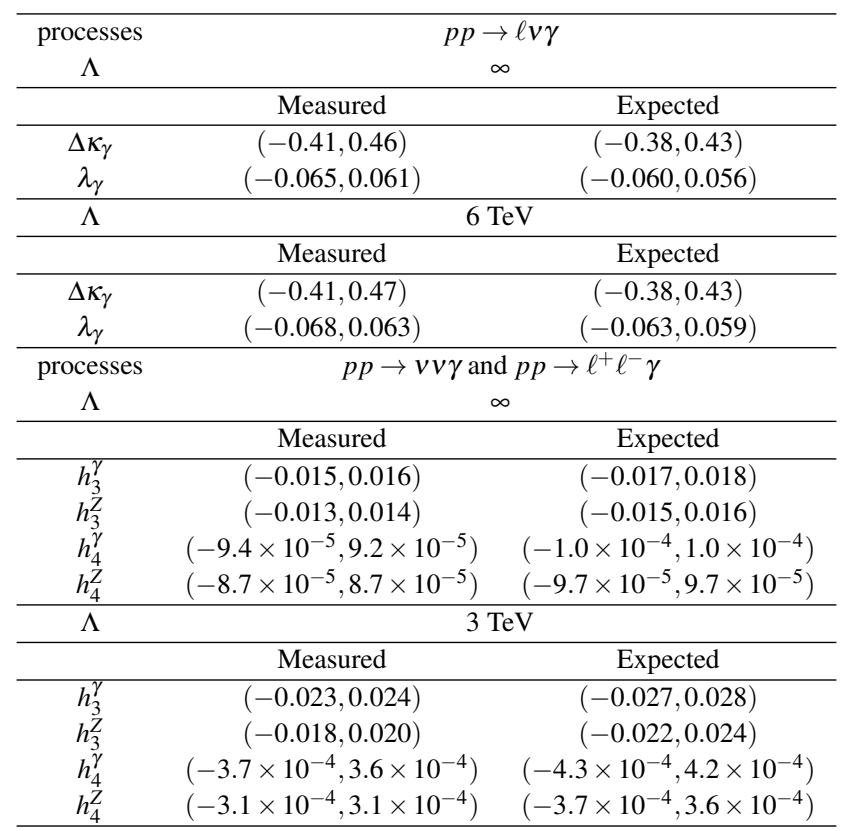

Table 4: The measured and expected 95\% CL intervals on the charged $\left(\Delta \kappa_{\gamma}, \lambda_{\gamma}\right)$ and neutral $\left(h_{3}^{\gamma}, h_{3}^{Z}, h_{4}^{\gamma}, h_{4}^{Z}\right)$ anomalous couplings. Results obtained using different $\Lambda$ values are shown while all other couplings are set to the SM values.

\subsection{Narrow resonance search}

New physics may also appear in the form of resonant states. Models such as Low Scale Technicolor [9] (LSTC) predict spin-1 mesons $\left(\omega_{\top} \rightarrow Z \gamma\right.$ and $\left.a_{\top} \rightarrow W \gamma\right)$ that have significant branching ratios to $W \gamma$ and $Z \gamma$. Since the width of the resonances predicted by these models is much smaller than the detector resolution, they are used as a benchmark to look for the presence of a resonance in the $W \gamma$ and $Z \gamma$ final states. In this study, only the $\left(\ell^{+} \ell^{-} \gamma\right)$ and the $(\ell v \gamma)$ final states are used. The signal comes from a resonance decaying into either the $W \gamma$ or $Z \gamma$ final states, so its decay products are boosted. Therefore in order to increase the exotic signal over SM background ratio, the cut on the photon transverse energy is raised to $E_{\mathrm{T}}^{\gamma}>40 \mathrm{GeV}$. No requirement is made on the jet multiplicity in this search. The exotic resonance signals as well as the SM backgrounds are modeled using probability density functions.

In the $\ell^{+} \ell^{-} \gamma$ final state, the search is conducted on the three-body invariant mass $m^{\ell \ell \gamma}$ distribution. The signal is modeled by a function which is the sum of a Crystal-Ball and a Gaussian. The SM backgrounds are modeled by a double exponential function. In the $\ell v \gamma$ final state, the 
search is performed on the three-body transverse mass ${ }^{3}$ distribution. The signal is modeled by a Crystal-Ball function while the SM backgrounds are modeled by a double exponential function. The model signal+background is then fit to the data to test for the presence of new physics. The data are interpreted using a modified frequentist approach $\left(C L_{S}\right)$ for setting limits.

In order to provide generic results that can be compared to any type of model that predicts narrow resonances decaying to these final state, a limit on the fiducial cross-section is presented. The fiducial volume follows the definition introduced in Table 1, but with $E_{\mathrm{T}}^{\gamma}>40 \mathrm{GeV}$ instead. Figure 2 presents the limits obtained from the search for the (left) $\ell^{+} \ell^{-} \gamma$ and (right) $\ell v \gamma$ final states. Using the LSTC benchmark model, the production of $a_{\mathrm{T}}$ is excluded up to $m_{a_{\mathrm{T}}}=703 \mathrm{GeV}$ in the $\ell v \gamma$ final state and the production of $\omega_{\top}$ is excluded up to $m_{\omega_{\top}}=494 \mathrm{GeV}$ in the $\ell^{+} \ell^{-} \gamma$ final state. The results of the vector resonance search are the first ones reported for the study of the $\ell v \gamma$ final state and the most stringent in the $\ell^{+} \ell^{-} \gamma$ final state.
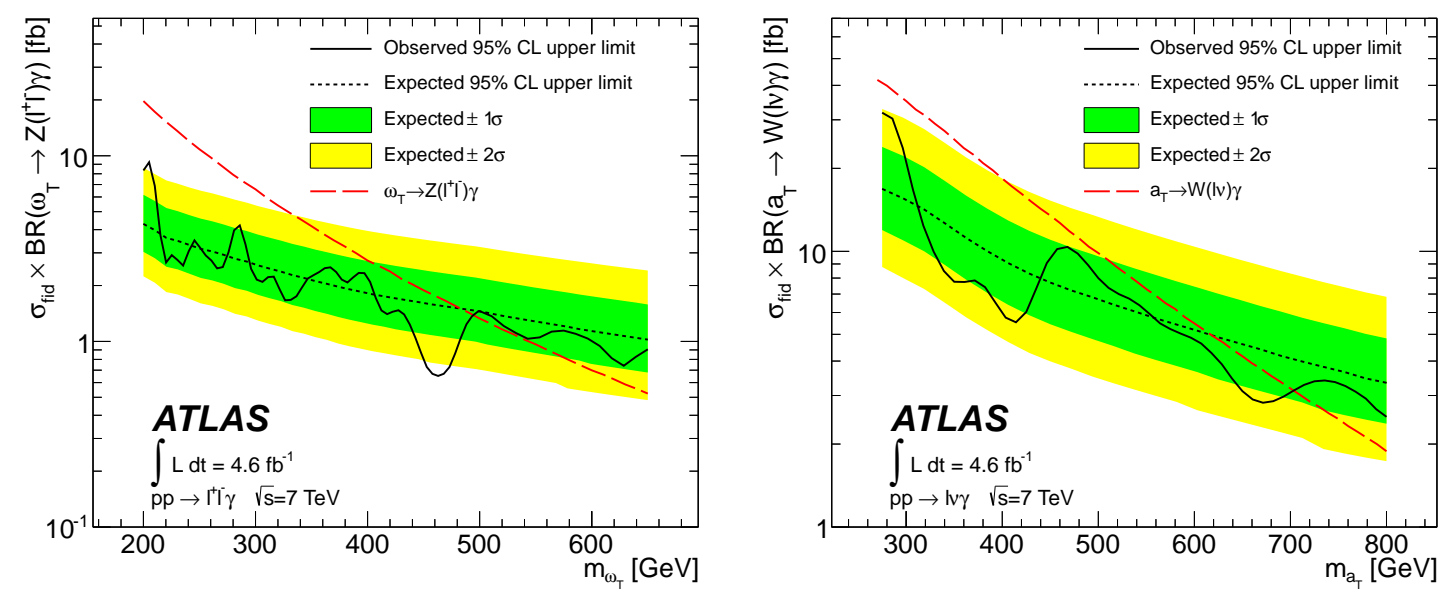

Figure 2: $95 \%$ CL limits [1] on narrow vector resonance production obtained using $\mathscr{L}=4.6 \mathrm{fb}^{-1}$ of data for the (left) $p p \rightarrow \ell^{+} \ell^{-} \gamma$ and (right) $p p \rightarrow \ell v \gamma$ final states.

\section{References}

[1] ATLAS Collaboration, Phys. Rev. D 87, 112003 (2013).

[2] U. Baur and E. L. Berger, Phys. Rev. D 41, 1476 (1990).

U. Baur, T. Han, and J. Ohnemus, Phys. Rev. D 48, 5140 (1993).

U. Baur and E. L. Berger, Phys. Rev. D 47, 4889 (1993).

[3] J. M. Campbell, R. Ellis and C. Williams, JHEP 1107, 018 (2011).

[4] H. -L. Lai et al., , Phys. Rev. D 82, 074024 (2010).

[5] T. Gleisberg et al., JHEP 0402, 056 (2004).

[6] M. L. Mangano et al., JHEP 0307, 001 (2003).

[7] P. M. Nadolsky et al., Phys. Rev. D 78, 013004 (2008).

[8] J. Pumplin et al., JHEP 0207, 012 (2002).

[9] K. D. Lane, Phys. Rev. D 60, 075007 (1999).

E. Eichten and K. Lane, Phys. Lett. B 669, 235 (2008).

\footnotetext{
${ }^{3}$ Three-body transverse mass: $\left(m_{\mathrm{T}}^{W \gamma}\right)^{2}=\left(\sqrt{m_{\ell \gamma}^{2}+\left|\vec{p}_{\mathrm{T}}(\gamma)+\vec{p}_{\mathrm{T}}(\ell)\right|^{2}}+E_{\mathrm{T}}^{\mathrm{miss}}\right)^{2}-\left|\vec{p}_{\mathrm{T}}(\gamma)+\vec{p}_{\mathrm{T}}(\ell)+\vec{E}_{\mathrm{T}}^{\mathrm{miss}}\right|^{2}$.
} 